\title{
Porous tantalum rods for treating osteonecrosis of the femoral head
}

\author{
Z.H. Liu, W.S. Guo, Z.R. Li, L.M. Cheng, Q.D. Zhang, D.B. Yue, \\ Z.C. Shi, B.L. Wang, W. Sun and N.F. Zhang \\ Department of Joint Surgery, China-Japan Friendship Hospital, Beijing, China \\ Corresponding author: W.S. Guo \\ E-mail: zhwscn@yeah.net
}

Genet. Mol. Res. 13 (4): 8342-8352 (2014)

Received August 5, 2013

Accepted March 21, 2014

Published October 20, 2014

DOI http://dx.doi.org/10.4238/2014.October.20.10

\begin{abstract}
This study evaluated the outcomes of using porous tantalum rods for the treatment of osteonecrosis of the femoral head $(\mathrm{ONFH})$. We performed core decompression and inserted porous tantalum implants in 149 patients (168 consecutive hips) with ONFH. Hips had large (65), medium (64), or small (39) lesions; 63 lesions were lateral, 68 were central, and 35 were medial. Conversion to total hip arthroplasty (THA) was the end point of this survey. A total of 130 cases (138 hips) were followed. The mean follow-up time was $38.46 \pm 5.76$ months; 43 hips (31\%) were converted to or needed THA. Of the 43 hips requiring THA, 33 had large lesions, including 1 medial, 3 central, and 29 lateral lesions; 9 had medium, lateral lesions, and 1 hip had a small, lateral lesion. Bone grafting was used in 59 hips, with 3 hips failing; 40 of 79 hips without bone grafts failed. The sum distances between the tops of the rods and the lateral lesion boundaries (SDTL, mm) were measured in anteroposterior and lateral radiographs. In the failure and spared groups, the average SDTLs were $7.65 \pm 2.759$ and $0.83 \pm 2.286 \mathrm{~mm}$, respectively. The survival of porous tantalum rods used for treating early-stage ONFH was affected by the size and location of the lesion, whether or not a
\end{abstract}


bone graft was used, as well as the distance between top of the rod and the lateral boundary of the lesion.

Key words: Porous tantalum rod; Osteonecrosis of the femoral head; Survival factors

\section{INTRODUCTION}

Nontraumatic osteonecrosis of the femoral head (ONFH) often occurs in young patients, continually troubling both the patient and the doctor. The disease, in many cases, progresses and negatively affects hip function, making hip replacement inevitable. However, the lifespan of young patients undergoing hip replacement exceeds the survival time of the primary prosthesis, making the long-term result of hip replacement less than ideal. Several preservative operations can be performed on the femoral head, which are intended to keep the femoral head intact and avoid or delay hip replacement. Porous tantalum rod implantation is a relatively new technique that has been widely applied to patients with early-stage osteonecrosis.

However, due to its use under several conditions (various sizes, locations), the clinical efficacy needs to be carefully evaluated. In our hospital, between January 2008 and June 2010, we performed core decompressions and insertions of porous tantalum rods for treating nontraumatic ONFH in patients with early-stage disease. This paper is based on an average 3-year follow-up, and describes the outcomes, clinical efficacy, and feasibility of porous tantalum rods for treating non-traumatic ONFH.

\section{MATERIAL AND METHODS}

All patients provided informed consent prior to participating in the study. All patients classified as having ONFH were diagnosed based on their clinical histories, physical examinations, and radiological evaluations by orthopedic surgeons in our department were studied. The stages and sizes of the ONFH lesions were based on the Association Research Circulation Osseous (ARCO) classification. The ONFH locations were measured according to the Japanese Investigation Committee of Health and Welfare Classification (Sugano et al., 2002). At the time of enrollment, 79 hips (47\%) were ARCO stage II and 89 (53\%) were ARCO stage III. Hips were determined to have large (65), medium (64), or small (39) lesions. The ONFH lesions were lateral (63 hips; type C-1, 21 hips, and type C-2, 42 hips), central (68 hips), or medial (35 hips). Patient outcomes were evaluated with routine methods, including limb-specific scores (Harris hip scores), radiographic outcome measures, and survivorship analyses. Any requirement for total hip arthroplasty (THA) was the end point of this survey. Contraindications included skin damage in the surgical region, active infection of the hip affected, clotting disorders, or anemia (hemoglobin $<100 \mathrm{~g}$, white blood cells $<4 \times 10^{9}$ ). All patients underwent anteroposterior and frog-position radiography, as well as magnetic resonance imaging and computed tomography (CT) of the hip(s) affected, preoperatively.

\section{Surgical techniques}

The operations were performed by five senior surgeons. Each patient was placed in 
supine position on a radiofluoroscope table, prepared for surgery, and received a 4-7-cm lateral hip incision. The iliotibial band was split, as was the vastus lateralis, along the incision line, to expose the lateral proximal femur. Under fluoroscopic guidance, a manufacturer-specified femoral guide pin hole was drilled from the proximal lateral femur into the $\mathrm{ONFH}$, and reamed along the path of the guide pin. After measuring the length of the implant, a measured porous tantalum rod (Zimmer, Warsaw, IN, USA), with a length of $80-95 \mathrm{~mm}$, a $10 \mathrm{~mm}$ diameter, and $14 \mathrm{~mm}$ threads, was inserted. In some patients, curettage along the tunnel to the subchondral bone was required to remove necrotic tissue within the anterolateral femoral head. Autologous bone was curetted from the ipsolateral iliac bone, mixed with $5 \mathrm{~g}$ of demineralized bone matrix and bone morphogenetic protein-rich demineralized bone matrix (Wright Medical Technology, Arlington, TN, USA) and layered into the cavity of the femoral head, prior to inserting the appropriate length porous tantalum rod.

\section{Observation and evaluation}

All patients were allowed to perform weight-bearing walking 12 weeks after the procedure, and hip joint functional exercises were encouraged. All preoperative Harris scores and images (ARCO staging) were recorded. During the follow-up, anteroposterior and frogposition radiographic films were examined; if necessary, CT was also performed. Clinical evaluations were scored based on the Harris scores. A score of $\geq 85$ was good, 70-85 was fair, and $<70$ was poor; Harris scores $\geq 70$ were defined as clinical success. Patients undergoing joint replacement surgery were considered clinical failures.

Anteroposterior and lateral radiographs were taken at a standard magnification of $115 \%$. Femoral head collapse progressing past ARCO stage III or to ARCO stage III from ARCO stage II, and joint space narrowing were regarded as radiographic failures. We also recorded the sizes and locations of the lesions. According to the ARCO system and the criteria of the Japanese Investigation Committee of Health and Welfare, a lesion occupying $<15 \%$ of the femoral head was classified as small, those occupying 15-30\% were medium, and those occupying $>30 \%$ of the femoral head were large. We also assessed the sum distance between the rod tip and the lateral lesion boundary (SDLT) by examining the anteroposterior and lateral films of the hip affected. The tip of the rod appears convex in the radiographs; therefore, we measured the shortest distance from the longitudinal line, parallel to the torso, through the lateral boundary of the lesion to the lateral edge of the rod tip.

\section{Statistical methods}

Measurement data are reported as means \pm standard deviation. The SPSS statistical software package, ver. 13.0 (IBM, Armonk, NY, USA) was used to calculate the means and to perform the Cox hazard model analysis, logistic regression analysis, and the Kaplan-Meier analysis. $\mathrm{P}<0.05$ was considered to be statistically significant.

\section{RESULTS}

A total of 130 patients (138 hips) were followed, including 69 patients ( 75 hips) with steroid-related ONFH, 37 cases (39 hips) with alcohol-related ONFH, and 24 patients (24 
hips) with idiopathic ONFH (Table 1). Thus, the largest proportion of patients had steroidrelated osteonecrosis (69\%). Despite the different ONFH etiologies, there were no significant differences among the cases with respect to preoperative hip function, or the degree of postoperative joint function improvement. A total of 47 hips (33.8\%) were classified as radiographic failures. The rate of imaging progression of pre-collapse hips was small $(29.7 \%)$, whereas the progression rate of post-collapse hips was $37.3 \%$.

\begin{tabular}{|c|c|c|c|c|}
\hline & Clinical success & Clinical failure & $P$ value & $95 \% \mathrm{CI}$ \\
\hline \multirow{2}{*}{\multicolumn{5}{|c|}{ Gender }} \\
\hline & & & & \\
\hline Male & 40 & 21 & & \\
\hline Female & 48 & 19 & & \\
\hline \multicolumn{5}{|l|}{ Etiology } \\
\hline Steroid-related & 51 & 24 & & \\
\hline Alcohol-related & 29 & 10 & & \\
\hline Idiopathic & 15 & 9 & & \\
\hline \multicolumn{5}{|l|}{ Preoperative Harris score } \\
\hline 0 & 31 & 12 & & \\
\hline 1 & 46 & 15 & & \\
\hline 2 & 18 & 16 & & \\
\hline \multicolumn{5}{|l|}{ Preoperative stage } \\
\hline ARCO II & 46 & 17 & & \\
\hline ARCO III & 49 & 26 & & \\
\hline \multicolumn{5}{|l|}{ Size } \\
\hline Small & 33 & 1 & & \\
\hline Medium & 41 & 9 & & \\
\hline Large & 21 & 33 & & \\
\hline \multicolumn{5}{|l|}{ Location } \\
\hline Medial & 28 & 1 & & \\
\hline Central & 56 & 3 & & \\
\hline Lateral & 11 & 39 & & \\
\hline Type C-1 & 11 & 11 & & \\
\hline Type C-2 & 0 & 28 & & \\
\hline Postoperative SDTL* $(\mathrm{mm})$ & $0.83 \pm 2.286$ & $7.65 \pm 2.759$ & $\mathrm{P}=0.000 ; \mathrm{F}=0.380$ & $5.932,7.707$ \\
\hline \multicolumn{5}{|l|}{ Bone grafting } \\
\hline Yes & 44 & 35 & & \\
\hline No & 51 & 8 & & \\
\hline
\end{tabular}

The follow-up time ranged from 29 to 50 months (38.46 \pm 5.76 months). A total of 19 patients (30 hips; 11 patients with bilaterally affected hips and 8 patients with unilateral lesions) were lost to follow-up. Among these cases, 12 ONFHs were at ARCO stage II and 18 were at ARCO stage III at the time of enrollment. For the unilaterally affected hips, the mean operative time was 1.1 hours and the blood loss was approximately $115 \mathrm{~mL}$. The clinical data showed that the average age of patients was 33.43 years (range, 20-48 years). Overall, the mean preoperative Harris score was 62.65 and the mean postoperative score was 79.50, showing a statistically significant improvement of 17 points $(\mathrm{P}<0.05)$. The results show an overall clinical score of "good" among $68.8 \%$ of the patients who were not lost to follow-up. If we assume that the 19 patients [ 30 hips, including 11 hips with large lesions (6 lateral, 4 central and 1 medial lesion), 14 hips with medium lesions (5 lateral, 4 central, and 5 medial lesions), and 
5 hips with small lesions (3 lateral and 2 central)] lost to follow-up were all clinical failures, the clinical success rate was $56 \%$. However, most of failures involved large or lateral lesions. If only the hips with large or lateral lesions in the lost to follow-up group were assessed as failures, the rate of clinical success was $63.1 \%$. In preoperative ARCO stage II patients, the clinical success rate was $74 \%$, and $61 \%$ in preoperative ARCO stage III patients.

Using conversion to THA as the end point of this survey, the rod survival rate was $95 \%$ after 1 year, $78 \%$ after 2 years, $72 \%$ after 3 years, and $68.8 \%$ after 4 years, suggesting that most of the clinical failures appeared within the first 2 postoperative years. As most progression accidents occurred in the large and lateral lesions groups, we analyzed the survival function in both groups (Figures 1 and 2) using a Kaplan-Meier analysis. In the large lesion group, hips with lateral lesions were more prone to failure, especially the type C-2 lesions. In the lateral lesion group, hips with large lesions had a greater tendency to fail (Figures 3-8).

\section{Survival function}

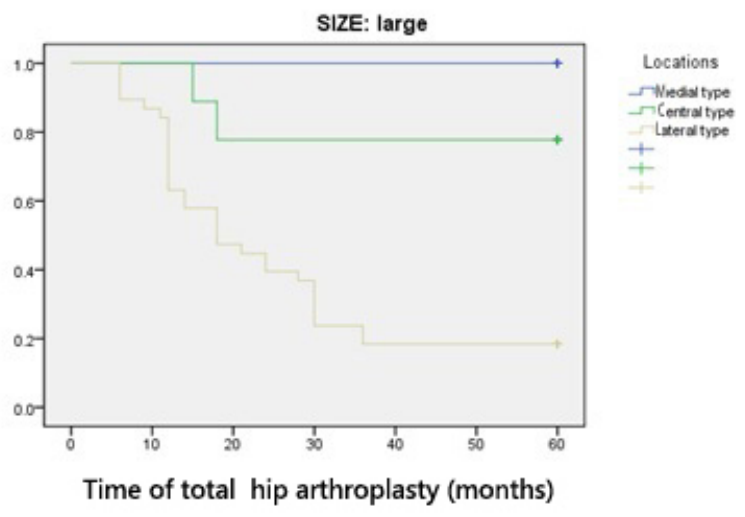

Figure 1. Kaplan-Meier survivorship analysis of the large lesion group. Hips with lateral lesions were more prone to failure, especially the type C-2 lesions.

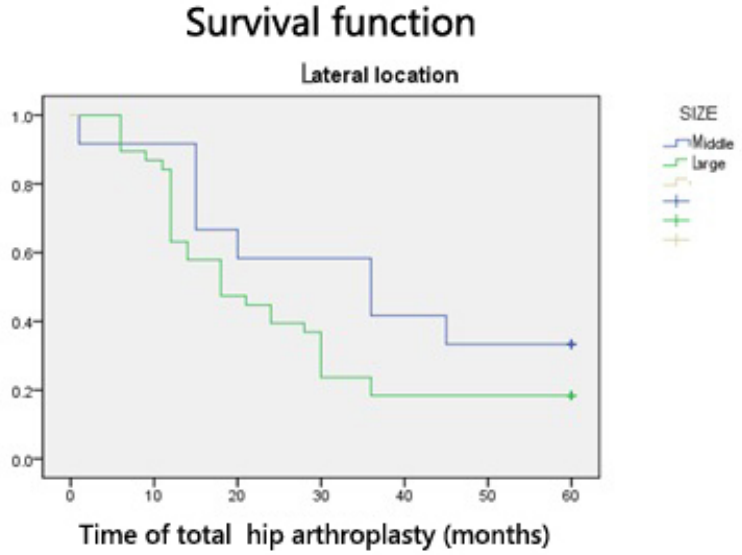

Figure 2. Kaplan-Meier survivorship analysis of the lateral lesion group. Hips with large lesions were more likely to fail. 


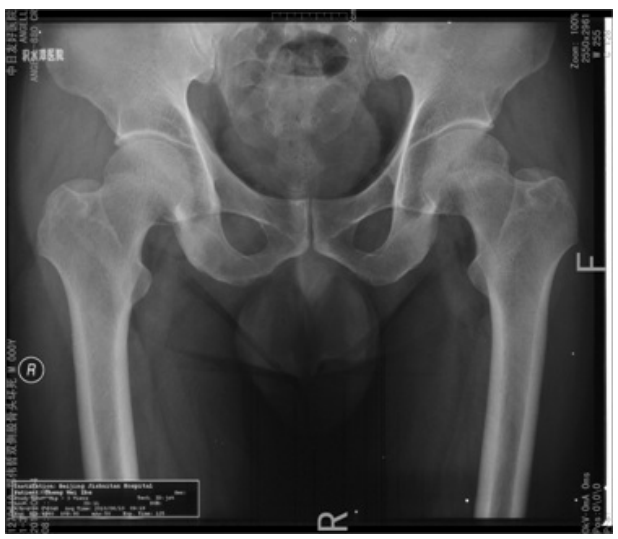

Figure 3. A 45-year-old man with bilateral osteonecrosis of the femoral head. The left side is Association Research Circulation Osseous (ARCO) stage II, showing large, lateral, type C-2 lesions; the right side is ARCO stage II, with small, central, type B lesions.

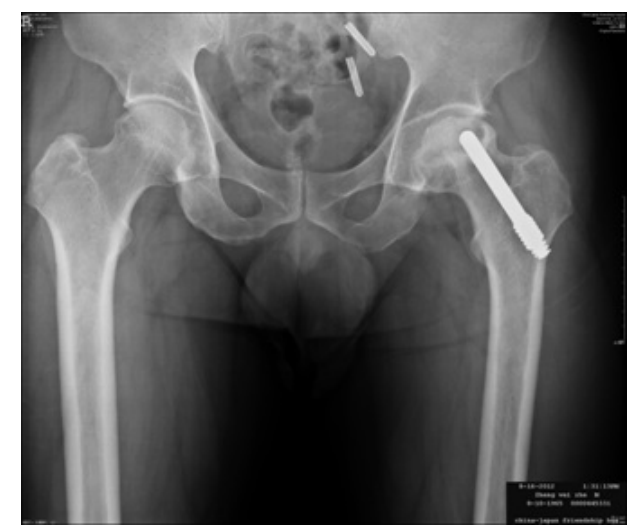

Figure 4. Left side was implanted with a bone graft and a porous tantalum rod.

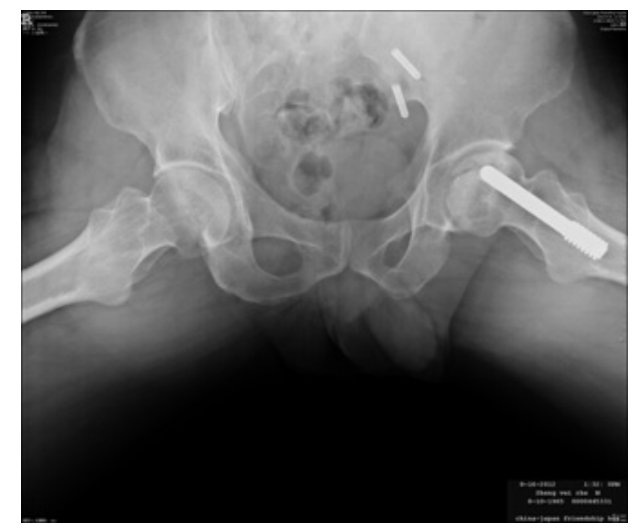

Figure 5. Two years later, the left femoral head collapsed and the region at the junction of the necrotic bone with living bone demonstrated subchondral bone fracture; the sum of the distances between the tops of the rods and the lateral lesion boundaries measured $>8 \mathrm{~mm}$. 


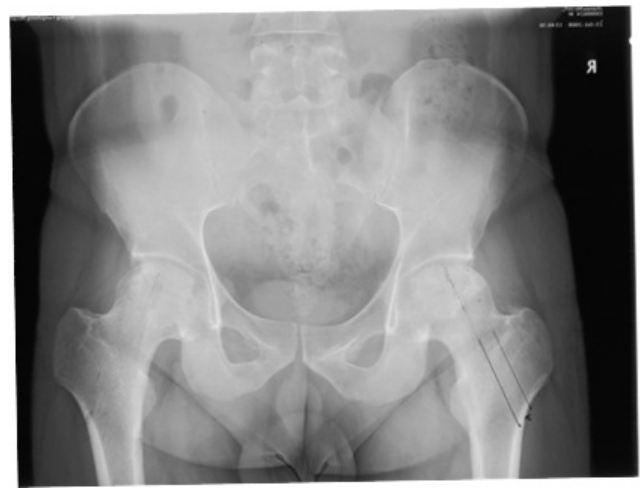

Figure 6. Anteroposterior radiographs of a 32-year-old man with bilateral osteonecrosis of the femoral head. The right side is Association Research Circulation Osseous (ARCO) stage IIIa (collapse of $<2 \mathrm{~mm}$ ), with large, lateral, type C-1 lesions; the left side is ARCO stage II, with large, lateral, type C-1 lesions. The template mark is drawn on the right side.

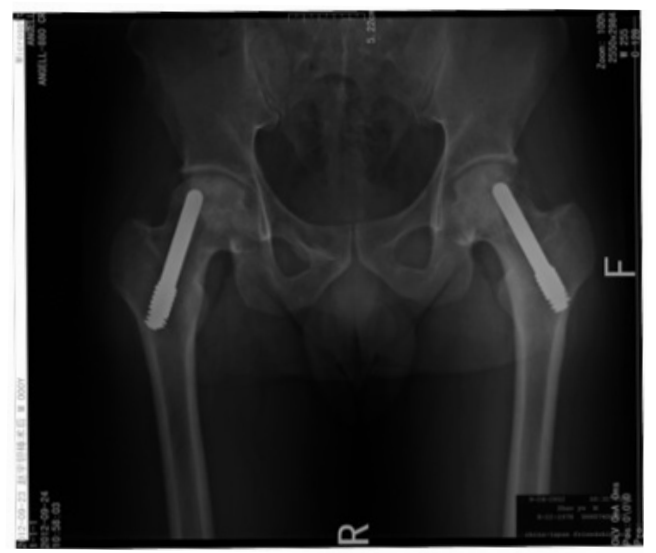

Figure 7. Four years later, both sides remained intact and new bone growth is seen within the previously necrotic region.

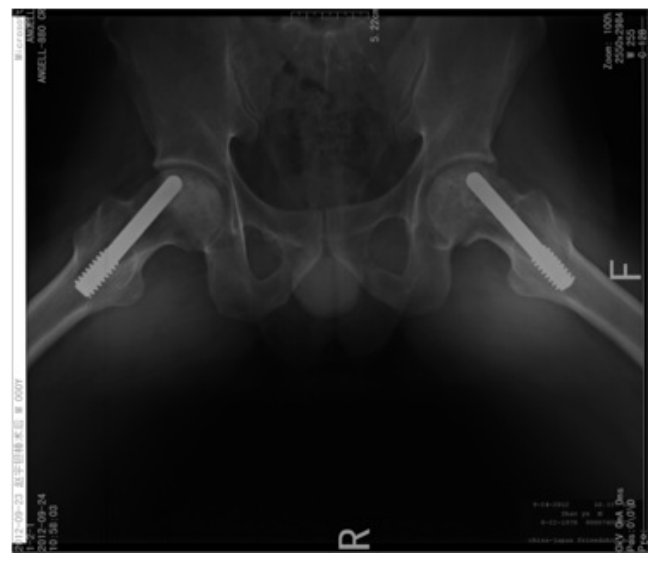

Figure 8. Both sides were implanted with bone grafts and porous tantalum rods; the sum distances between the tops of the rods and the lateral lesion boundaries measured $<1 \mathrm{~mm}$ on both sides. 
The average SDTL in the failure group was $7.65 \pm 2.759 \mathrm{~mm}$ and $0.83 \pm 2.286 \mathrm{~mm}$ in the non-failure group; the difference was significant. Among the failed hips, 35/79 (44.3\%) did not undergo bone-grafting, whereas only $8 / 59(13.6 \%)$ of those undergoing bone grafting failed (chi-square test, $\mathrm{P}=0.00$ ).

We grouped ONFHs according to lesion size, location, use of bone grafting, and conducted a Cox hazard model analysis that showed that lateral lesions $[\mathrm{P}=0.01$; degree of risk, 2.504; 95\% confidence interval (CI), 1.422-4.410] and SDLT ( $\mathrm{P}=0.001$; degree of risk, 0.467; $95 \% \mathrm{CI}=0.302-0.721)$ were independent risk factors for imaging failures. Lesion size $(\mathrm{P}=$ 0.031 ; degree of risk, 3. 085; 95\% $\mathrm{CI}=1.109-8.576)$, lesion location $(\mathrm{P}=0.010$; degree of risk, $2.287 ; 95 \% \mathrm{CI}=1.222-4.276)$, bone grafting $(\mathrm{P}=0.005$; degree of risk, $0.243 ; 95 \% \mathrm{CI}=0.091$ $0.647)$ and SDLT $(\mathrm{P}=0.005$; degree of risk, 0.534; 95\%CI $=0.346-0.824)$ were independent risk factors for clinical failure. However, clinical and radiological failures were not correlated with ONFH etiologies, presence of lesions within the femoral head epiphysis, gender, age. Logistic regression parameters and estimates for clinical success were calculated (Table 2).

Table 2. Logistic regression parameters for clinical success.
\begin{tabular}{lcccrc}
\hline Variable selected & $\mathrm{b}$ & $\mathrm{Sb}$ & Wald $\chi^{2}$ & $\mathrm{P}$ & OR \\
\hline Constants & -13.997 & 4.351 & 10.350 & 0.001 & 0.004 \\
Location of lesion & 6.615 & 2.270 & 8.491 & 0.04 & 746.508 \\
Bone graft & -6.475 & 2.452 & 6.975 & 0.002 \\
SDLT $(\mathrm{mm})$ & 1.763 & 0.623 & 8.017 & 5.829 \\
\hline
\end{tabular}

Regression equation is: $\ln (\mathrm{P} / 1-\mathrm{P})=-13.997+6.615$ (location) -6.475 (bone graft) +1.763 (SDLT). Clinical success was related to the lesion location, use of bone graft, and SDLT. SDLT, the sum distance between the lateral edge of rod head and lateral boundary of lesion on anterioposterior and lateral films, measuring the shortest distance from the longitudinal line parallel to the torso, through the lateral boundary of lesion, to the line through the lateral edge of the tip. Both $\mathrm{b}$ and $\mathrm{sb}$ represent variable constants in logistic regression equation $\mathrm{n}$ the SPSS software system.

\section{DISCUSSION}

ONFH mainly affects young, active individuals. The ideal way to treat this disease is to reduce pain, keep the femoral head round, and prevent or delay the deterioration of hip function. The current head-preserved surgical treatments of ONFH involve core decompression, debridement plus bone implantation including structural and non-structural bone grafts. (Rosenwasser et al., 1994). Especially for the non-structural bone grafts, various types of bone grafts have been developed to repair the structure of the femoral head and prevent its collapse, prevent secondary osteoarthritis, and delay joint replacement surgery in young patients. Moreover, these also affect future joint replacement surgeries, but to a lesser extent (Mont et al., 2003; Aldridge and Urbaniak, 2004; Steinberg et al., 2001, 2008).

After ONFH decompression, structural support problems arise. Urbaniak in the 1970s and 1980s, began to use vascularized bone graft technology to improve the success rate of bone transplantation in ONFH patients (Urbaniak et al., 1995). Since then, a large number of cases have confirmed the effectiveness of this technology through follow-up periods (Buckley et al., 1991). Aldridge et al. (2004) applied free vascularized fibular grafts in patients with collapsed ONFHs. Their clinical success rate, reported for 188 cases (224 hips) with an average follow-up of 4.3 years, was $67.4 \%$ after 2 years, $64.5 \%$ after 5 years, and $63 \%$, overall. Kawate et al., (2007) reported a 7 -year success rate of $67 \%$, and indicated that the success 
rate in patients with preoperative, pre-collapsed ONFH was significantly higher than that in patients with preoperative, collapsed ONFH. However, vascularized bone graft surgery is a long, complex surgery that is more traumatic and subject to significant complications, such as peroneal nerve palsy, post-transplantation subtrochanteric fracture, and ankle pain. The use of non-vascularized bone grafting is, therefore, more common than the use of vascularized bone grafts. Rather than using vascularized bone grafts, the use of additional additives, such as bone graft substitutes, bone growth factors, etc., have demonstrated improved results (Rijnen et al., 2003; Keizer et al., 2006; Deirmengian et al., 2008).

Porous tantalum rod implantation for treating ONFH is a newer, alternative technology. The porous tantalum rods are believed to have better physiological stress distribution, and can provide good structural support in the necrotic femoral head area. The initial application of this technology for treating ONFH also considered its elastic modulus, which is similar to bone, and the interspace within the rod is favorable for bone conduction (Bobyn et al., 2004; Tsao et al., 2005).

Our department is one of the first units in China to adopt this technique for treating ONFH. In our study, the use of porous tantalum rods in the reconstruction of femoral heads, in the short term, seemed acceptable; the overall clinical success rate was $63.1-68.8 \%$ (59\% radiographic success rate). In ARCO stage II patients, preoperatively, the success rate was $74 \%$, and $61 \%$ in ARCO stage III patients. We found that, in some cases, after the tantalum rod was implanted, the femoral head continued to collapse, leading to early failures. However, in other cases the femoral head could be supported by the tantalum rod for a long time. Clinical comparative analyses suggested that the tantalum rod could be used for treating ONFH, but particular attention needed to be given to its indications (Kim et al., 2010; Floerkemeier et al., 2011; Liu et al., 2012). From our experience treating ONFH with porous tantalum rods, regression analyses showed that the clinical success rate was related to the preoperative size and location of the lesion, whether or not bone grafting was used, and the SDTL. We also found that the simple insertion of the porous tantalum rod had a significant failure rate. Tanzer et al. (2008) also reported 17 cases of tantalum rod failure, and suggested that single tantalum rod in the necrotic zone may be limited to conduct the formation of new bone and have a limited support to subchondral bone.

The studies above showed that one of the critical factors for the support playing a long-term role in ONFH lesions is the recovery of blood supply around the support. Our research also suggested that supports placed in ONFH lesions are, importantly, prone to stress fractures (Brown et al., 1992). Motomura et al. (2011) reported a series of collapsed ONFH lesions that demonstrated stress fractures in the lateral region of the lesion. Moreover, an earlier study of ONFH pathology also suggested that the region at the junction of necrotic bone with living bone appeared to be characterized by thinning subchondral bone, possibly due to tissue absorption (Glimcher and Kenzora, 1979). In addition to the support of the necrotic femoral head lesions, the removal of dead bone and bone grafting at the junction with living bone is important for surgical success. Tantalum rods provide a limited support area $\left(<1 \mathrm{~cm}^{2}\right)$; therefore, removal of the subchondral bone lesion and bone grafting has an important impact. In type $\mathrm{C}-2$ lesions, tantalum rods may not provide adequate support at the junction between necrotic and living bone zones.

On the other hand, we recommend that bone grafts should be implanted in the necrotic area and make contact with the living bone zone since the porous rod only plays a support role during the early stages of healing. Because of the involvement of intraoperative debride- 
ment and bone grafting in the technique, there is a risk of damaging the subchondral bone and articular cartilage. Therefore, the volume and depth of the debridement should be strictly controlled. Our experience indicates that debridement should extend 1-2 mm under the subchondral plate, guided by the $\mathrm{C}$-arm fluoroscope. Complete removal of the necrotic bone should be avoided to prevent reducing the femoral head's mechanical strength. During insertion of the bone graft, strength should be moderate and the compression should be layer by layer to avoid building up the articular cartilage from the top. Bone graft donor site complications have been reported in many studies. However, we have taken autograft bone from the iliac bone and the process has proven to be safe and effective. We believe that autologous bone transplantation and the use of composite demineralized bone matrix provide satisfactory results.

We presume that the design of the porous tantalum rods could be improved and that more common metals, e.g., titanium, could be alternative materials for use in the fixed part of the rod - the threaded end and the smooth part. As currently used in revision surgeries for failed porous tantalum rods, removal of these failed devices is troublesome (in non-necrotic area of bone, due to bone ingrowth, the fixed part of tantalum rod has a close contact with live bone).

In conclusion, we believe that the porous tantalum rod insertion technique, in conjunction with debridement of the necrotic ONFH lesion and bone implantation, should be suitable for patients selected with early-stage ONFH lesions. Before surgery, the templated tip of the inserted rod should be close to the lateral boundary of the lesion on the anteroposterior and lateral films; otherwise, the rod should be abandoned. For ONFH lesions that are large and laterally located, caution should be exercised in the selection of the porous tantalum rod technique, especially for type C-2 ONFH lesions.

\section{REFERENCES}

Aldridge JM III and Urbaniak JR (2004). Bone grafting for osteonecrosis of the femoral head. Semin. Arthroplasty 15: 151-160.

Aldridge JM III, Berend KR, Gunneson EE and Urbaniak JR (2004). Free vascularized fibular grafting for the treatment of postcollapse osteonecrosis of the femoral head. Surgical technique. J. Bone Joint Surg. Am. 86-A (Suppl): 1: 87-101.

Bobyn JD, Poggie RA, Krygier JJ, Lewallen DG, et al. (2004). Clinical validation of a structural porous tantalum biomaterial for adult reconstruction. J. Bone Joint Surg. Am. 86-A (Suppl 2): 123-129.

Brown TD, Baker KJ and Brand RA (1992). Structural consequences of subchondral bone involvement in segmental osteonecrosis of the femoral head. J. Orthop. Res. 10: 79-87.

Buckley PD, Gearen PF and Petty RW (1991). Structural bone-grafting for early atraumatic avascular necrosis of the femoral head. J. Bone Joint Surg. Am. 73: 1357-1364.

Deirmengian GK, Israelite CL, Nelson CL and Garino JP (2008). Bone grafting procedures. Techn. Orthopaed. 23: 35-43.

Floerkemeier T, Lutz A, Nackenhorst U, Thorey F, et al. (2011). Core decompression and osteonecrosis intervention rod in osteonecrosis of the femoral head: clinical outcome and finite element analysis. Int. Orthop. 35: 1461-1466.

Glimcher MJ and Kenzora JE (1979). The biology of osteonecrosis of the human femoral head and its clinical implications: II. The pathological changes in the femoral head as an organ and in the hip joint. Clin. Orthop. Relat. Res. 283-312.

Kawate K, Yajima H, Sugimoto K, Ono H, et al. (2007). Indications for free vascularized fibular grafting for the treatment of osteonecrosis of the femoral head. BMC Musculoskelet. Disord. 8: 78.

Keizer SB, Kock NB, Dijkstra PD, Taminiau AH, et al. (2006). Treatment of avascular necrosis of the hip by a nonvascularised cortical graft. J. Bone Joint Surg. Br. 88: 460-466.

Kim JO, Roh KJ, Park HS, Sohn HS, et al. (2010). The 5 year follow-up use of a tantalum trabecular metal system for early stage of osteonecrosis of the femoral head. J. Bone Joint Surg. Br. 92: 1-138.

Liu B, Sun W, Yue D, Li Z, et al. (2013). Combined tantalum implant with bone grafting for the treatment of osteonecrosis of the femoral head. J. Invest. Surg. 26: 158-162.

Mont MA, Etienne G and Ragland PS (2003). Outcome of nonvascularized bone grafting for osteonecrosis of the femoral head. Clin. Orthop. Relat. Res. 84-92. 
Motomura G, Yamamoto T, Yamaguchi R, Ikemura S, et al. (2011). Morphological analysis of collapsed regions in osteonecrosis of the femoral head. J. Bone Joint Surg. Br. 93: 184-187.

Rijnen WH, Gardeniers JW, Buma P, Yamano K, et al. (2003). Treatment of femoral head osteonecrosis using bone impaction grafting. Clin. Orthop. Relat. Res. 74-83.

Rosenwasser MP, Garino JP, Kiernan HA and Michelsen CB (1994). Long term followup of thorough debridement and cancellous bone grafting of the femoral head for avascular necrosis. Clin. Orthop. Relat. Res. 17-27.

Steinberg ME, Larcom PG, Strafford B, Hosick WB, et al. (2001). Core decompression with bone grafting for osteonecrosis of the femoral head. Clin. Orthop. Relat. Res. 71-78.

Steinberg ME, Lai M, Garino JP, Ong A, et al. (2008). A comparison between total hip replacement for osteonecrosis and degenerative joint disease. Orthopedics 31: 360 .

Sugano N, Atsumi T, Ohzono K, Kubo T, et al. (2002). The 2001 revised criteria for diagnosis, classification, and staging of idiopathic osteonecrosis of the femoral head. J. Orthop. Sci. 7: 601-605.

Tanzer M, Bobyn JD, Krygier JJ and Karabasz D (2008). Histopathologic retrieval analysis of clinically failed porous tantalum osteonecrosis implants. J. Bone Joint Surg. Am. 90: 1282-1289.

Tsao AK, Roberson JR, Christie MJ, Dore DD, et al. (2005). Biomechanical and clinical evaluations of a porous tantalum implant for the treatment of early-stage osteonecrosis. J. Bone Joint Surg. Am. 87 (Suppl 2): 22-27.

Urbaniak JR, Coogan PG, Gunneson EB and Nunley JA (1995). Treatment of osteonecrosis of the femoral head with free vascularized fibular grafting. A long-term follow-up study of one hundred and three hips. J. Bone Joint Surg. Am. 77: 681-694. 